\title{
QOL-associated factors in elderly patients who underwent cardiovascular surgery
}

\author{
Kiyoko Terashima $\cdot$ Yoko Yoshimura • \\ Kazuyoshi Hirai $\cdot$ Yukinori Kusaka
}

Received: 17 February 2010/Accepted: 13 July 2011/Published online: 13 August 2011

(C) The Japanese Society for Hygiene 2011

\begin{abstract}
Objectives Quality of life (QOL) was compared between elderly patients treated with cardiac surgery (cardiac surgery group) and general elderly nonoperated individuals (control group) to clarify differences in QOL-associated factors between the two groups.

Methods The cardiac surgery group consisted of 82 patients aged 72.5 years at approximately 2 years after discharge, and the control group consisted of 95 elderly individuals aged 77 years living at home. QOL was measured based on interviews using two scales: disease-specific QOL and Short Form (36) Health Survey (SF-36) in the cardiac surgery group, and SF-36 in the control group. Associated factors included the Tokyo Metropolitan Institute of Gerontology (TMIG) index of competence, living with one's spouse, exercise habit, and the number of past medical history items.

Results There was no cardiac surgery procedure-associated significant difference in disease-specific QOL. When comparing SF-36 between groups, the physical health level was significantly lower in the cardiac surgery group, but no significant difference was noted in the mental health level. Regarding physical health level of SF-36-associated factors, mental health level was a negative factor in both groups. In the cardiac surgery group, age was a negative
\end{abstract}

K. Terashima $(\bowtie) \cdot$ Y. Yoshimura

Faculty of Nursing and Social Welfare Sciences,

Fukui Prefecture University, 4-1-1 Kenzyozima,

Matsuoka, Eiheizi-cho, Fukui 910-1195, Japan

e-mail: terasima@fpu.ac.jp

K. Hirai · Y. Kusaka

Department of Environmental Health, School of Medicine,

University of Fukui, 23-3 Shomoaitsuki, Matsuoka,

Eiheizi-cho, Fukui 910-1193, Japan factor. In the control group, the TMIG index and number of past medical history items were positive and negative factors, respectively.

Conclusions As elderly people with high physical health levels after cardiac surgeries may have low mental health levels, physicians and nurses need to be aware and address this situation.

Keywords Cardiovascular surgery $\cdot$ Disease-specific QOL - SF-36 - TMIG index of competence .

Comparison study

\section{Introduction}

The mortality rate from chronic diseases has increased with the aging of society, and the number of deaths from heart diseases in 2006 in Japan was 173,125, accounting for $16.0 \%$ of all deaths. Mortality rates (per 100,000 persons) from ischemic heart disease in men and women were $68.1 \%$ and $53.5 \%$, respectively, accounting for $44.3 \%$ of all deaths from heart diseases [1]. The number of surgeries performed in the cardiac surgery field has been increasing over the years, from 20,000 in the late 1980 s to 54,000 in 2006, and surgeries for ischemic heart disease accounted for about one third of all cardiac surgeries. According to a survey performed by the Japanese Association for Coronary Artery Surgery, coronary artery bypass grafting (CABG) was performed in 14,583 cases at 319 facilities nationwide in 2007, and an off-pump procedure using a nopump oxygenator, established as the standard CABG procedure, was employed in more than 60\%, [2], indicating that low-invasive cardiovascular surgery has been rapidly spreading and the number of aging patients undergoing surgery will further increase. 
However, surgery of "the heart," an organ that strongly symbolizes life, may have a marked psychological influence on elderly patients. Moreover, elderly people's conditions become complicated by the impairment of motor organs with aging, which makes it difficult to maintain activity during the postoperative process of discontinuing bed rest and after discharge. Therefore, the quality of life (QOL) of elderly patients following cardiac surgery may be influenced by many factors in a complex way in addition to the physical stress of surgery. The purpose of cardiovascular surgeries has changed to improved QOL from simply treatment and prolongation of life; for this purpose, QOL has been actively investigated [3-10]. However, most studies evaluate surgical indications based on the New York Heart Association (NYHA) and Canadian Cardiovascular Society (CCS) survival rate before and after surgery, the effect and treatment, and intervention employing a QOL scale only from viewpoints of health care workers. Cardiac surgery improves survival rate and symptoms and improves QOL in many reports [11], but there have been fewer reports on QOL evaluation from viewpoints of the elderly patients themselves, such as whether they found pleasure in and realized the significance of their own social roles.

In this study, participants were elderly patients at approximately 2 years after CABG, valve replacement, or aortic/thoracic aortic bypass grafting at the same medical institution who were relatively healthy and could continue outpatient visits. The time required after cardiac surgery to recover their preoperative QOL level markedly varies among patients, but the influence of the physical stress of surgery may decrease as the stay at home under outpatient treatment alone prolongs. To confirm this, we considered that a comparison of QOL between patients and general elderly individuals was necessary. We also believed it necessary to consider that psychosomatic functions decline with aging and as the duration of follow-up progresses, and serves as a QOL-influencing factor. Therefore, to clarify the actual state of QOL of elderly patients, we excluded the period immediately after discharge in which surgeryassociated physical stress may be marked and when agerelated changes are expected to exceed the influence of surgery.

Accordingly, the objective of this study was to compare QOL between elderly cardiac surgery patients and nonoperated elderly individuals by employing the overall QOL scale. We thus investigated differences in QOL-associated factors between groups to clarify the characteristics of QOL of elderly patients treated with cardiac surgery. Although this study did not aim at clarifying changes in their QOL based on the characteristic symptoms of the disease before and after surgery, it cannot be ruled out that diseases treated with surgery and the surgery itself influenced the overall QOL, and these influences may have been QOL-associated factors of elderly cardiac surgery patients. Thus, we concomitantly employed the diseasespecific QOL scale with an overall QOL scale.

\section{Methods}

\section{Participants}

Cardiac surgery patients (cardiac surgery group) were aged $\geq 65$ years or older out of 381 patients who underwent CABG, valve replacement, or aortic/thoracic aortic bypass grafting performed by the same circulatory surgeon between May 1996 and March 2002 at Fukui Prefectural Hospital, which performs the largest number of cardiac surgeries in Fukui Prefecture. Eighty-two of 226 outpatients within 2 months to 6 years after discharge consented to participate in the study after direct explanation of the study by investigator $\mathrm{T}$ (participation rate $36.2 \%$ ). For the control group, 100 second-year students at the Fukui Prefectural University Faculty of Nursing and Social Welfare each interviewed one individual aged $\geq 65$ years who were their grandparents or neighbors. The students were instructed to comply with the following conditions: individuals had not been hospitalized within 1 month before the interview day and could communicate sufficiently to answer the questions; the duration of the interview was no more than $30 \mathrm{~min}$. The response rate was $95.0 \%$.

\section{Survey method and period}

For the cardiac surgery group, 37 survey days were set between May 2002 and March 2003. The control group was surveyed in December 2001 and December 2002.

\section{Survey content}

There are two methods of QOL measurement: overall evaluation with no disease specificity; and specific scales designed to consider disease-specific contents. Different QOL issues are addressed in the two scale. Thus, we used both scales in this study.

\section{Disease-specific QOL scale}

This scale is used to closely measure disease-specific symptoms and influences developed for each disease. Compared with the overall evaluation with no disease specificity, this scale collects much information on symptoms characteristic to the diseases, and the measurement sensitivity of time courses changes when health conditions are high [12]. For the disease-specific QOL scale, we used 
the questionnaire on QOL and disease established by Kayaba et al. [13], for which the reliability and validity have been verified This questionnaire was developed to measure QOL in the general Japanese population. We also used disease-specific scales, because circulatory disease is a life-long condition. Patients are assessed based on three domains: disease-specific symptoms and pathological condition, nonspecific health condition, and social and subjective indices.

Diseases-specific symptoms and pathological condition We assessed ten items in this domain, for example, questions regarding ischemic heart disease: Do you have chest pain (or a compressed feeling of the chest) while climbing up steps in a station? and Do you get short of breath (or dyspnea) while bathing, defecating, or changing clothes?

Nonspecific health condition This is related to general health and not related to specific circulatory disease, from which eight items were assessed, including: Do you have palpitations or get short of breath? Are your hands and legs swollen?, and questions about appetite loss, insomnia, and fatigue.

Social and subjective indices We considered that questions in the social and subjective indices domain established by Kayaba et al. [13] are not appropriate for individuals $\geq 65$ years or older. Thus, we adopted subjective QOL questions used by the National Cardiovascular Center Study Group's 2A-4 Study on the management system for the aged patients with cardiovascular diseases to improve their quality of life [14]. There were 15 questions among five subdomains: (1) feeling of being a burden due to disease, (2) mental stability, (3) independence, (4) sense of satisfaction, (5) interest and activity in life.

Scoring Three possible responses to the three major domains are: yes, neither, and no, and rated 0,1 , and 2 points, respectively, so that higher points indicate a more favorable QOL and vice versa.

$S F-36$

For the overall QOL scale, the Japanese version of the Short Form (36) Health Survey (SF-36) questionnaire (Ver 1.2) [15] was used. This survey is composed of factors shared by patients with various diseases and general healthy persons: physical functioning (PF), role physical (RP), body pain (BP), general health $(\mathrm{GH})$, vitality (VT), social function (SF), role emotional (RE), and mental health $(\mathrm{MH})$. The first four subscales are physical component summaries (PCS); the remainder are mental component summaries (MCS). Several subscale questions were grading from second to fifth rank on a maximum scale of 100 points, so that a higher score indicates a more favorable QOL. The lowest possible PCS scores indicate limitations in self-care; in physical social, and role activities, severe bodily pain, and frequent tiredness. The highest possible score indicates no physical limitations, disabilities, or decrements in well-being and a high energy level. The lowest possible MCS score means frequent psychological distress, and social and role disabilities due to emotional problems. The highest possible score means frequent positive affect, absence of psychological distress, and limitations in usual social/role activities due to emotional problems [16].

Associated factors

As QOL-associated factors, gender, age, family composition, living with one's spouse, exercise habit, the number of past medical history items, and activity capacity were investigated. Regarding activity capacity, the Tokyo Metropolitan Institute of Gerontology (TMIG) index of competence [17] was employed. This index is a multidimensional scale composed of 13 items: five concerning instrumental independence, four concerning intellectual activity, and four concerning social role. We used these three subscales.

\section{Analytic method}

Scores of the three subscales of the disease-specific QOL scale, two summary scores of the physical (PCS) and mental (MCS) component summaries representing the physical and mental health levels on the SF-36, and scores of three subscales of the TMIG index were evaluated. For differences associated with surgical procedures, after confirming homogeneity, we used the $t$ test or Mann-Whitney $U$ test. Association of each factor with the disease-specific QOL scale and SF-36 and those between the two scales were analyzed with Spearman's rank correlation coefficient. To compare the TMIG index and SF-36 between the surgery and control groups, univariate analysis of variance (ANOVA) was used, with age and gender as covariates. For comparison of the other rates, the $\chi^{2}$ test and Fisher's direct probability method were used. To compare QOLassociated factors between the two groups, participants were divided into high- and low-score groups with PCS and MCS medians of SF-36 in the control group set as cutoff values. Associated factors surveyed, such as gender, age, family composition, living with one's spouse, exercise habit, number of past medical history items, and activity capacity, were considered for all as dependent variables, 
and stepwise multiple logistic regression analysis using the forward selection method was performed. The significance level was set at 5\%, and SPSS ver. 14.0 was used for statistical analysis.

\section{Results}

Comparison of associated factors and QOL by surgical procedure in the cardiac surgery group

Median duration of hospitalization in the cardiac surgery group was 45.0 (range 20-158) days, the median time after discharge was 772.5 (range 80-2,094) days, and more than half of the patients were surveyed 2 years or more after discharge. The applied cardiac surgery was CABG in 51 patients $(62.2 \%)$, valve replacement in seven $(8.5 \%)$, and aortic/thoracic aortic bypass grafting in 24 (29.3\%). Table 1 shows a comparison of associated factors by surgical procedure. The median time after discharge was shortest in the CABG group. All patients in the valve replacement group lived with their spouses, and the same group had the lowest rate of exercise habit. All patients had a past medical history of conditions other than heart disease. These associated factors and QOL scores were compared among the surgical procedure groups, but no significant differences were noted. Thus, all patients were collectively analyzed as the cardiac surgery group.

Association between disease-specific QOL and SF-36

The association between the two scales was analyzed using Spearman's correlation coefficient. Results are shown in Table 2. All scores of the disease-specific QOL scales were significantly correlated with MCS score at $r=0.5$ or higher, but no significant association with PCS was observed. It was clarified that the disease-specific QOL scale reflected MCS in SF-36.

Comparison of associated factors and SF-36 between cardiac surgery and control groups

We compared associated factors and PCS and MCS of SF36 between the cardiac surgery group consisting of 82
Table 1 Comparison of characteristics, Tokyo Metropolitan Institute of Gerontology (TMIG), diseasespecific quality of life (QOL), and the Short Form (36) Health Survey (SF-36) summary scores among the three surgeries

Gender, living with spouse, family structure and exercise habit values are expressed as no. Number of previous diseases including heart diseases is per person. Numbers shown in mean (SD) are for normal distributions, and others are in median

$C A B G$ coronary artery bypass graft, $P C S$ physical component summaries, $M C S$ mental component summaries, n.s. not significant

a Kruskal-Wallis test

b Chi-square test

${ }^{\mathrm{c}}$ Fisher test

\begin{tabular}{|c|c|c|c|c|}
\hline & CABG & Valve replacement & Aorta/thoracic & $p$ value \\
\hline Days after discharge & 687.0 & 806 & 970.1 & n.s. ${ }^{a}$ \\
\hline \multicolumn{5}{|l|}{ Gender } \\
\hline Male & 43 & 5 & 15 & \multirow[t]{2}{*}{ n.s. ${ }^{b}$} \\
\hline Female & 8 & 2 & 9 & \\
\hline Age & 72.0 & 70.0 & 73.0 & n.s. ${ }^{a}$ \\
\hline \multicolumn{5}{|l|}{ Living with spouse } \\
\hline Yes & 44 & 7 & 13 & \multirow[t]{2}{*}{ n.s. ${ }^{b}$} \\
\hline No & 7 & 0 & 11 & \\
\hline \multicolumn{5}{|l|}{ Family composition } \\
\hline Alone & 3 & 0 & 3 & \multirow[t]{3}{*}{ n.s. ${ }^{\mathrm{c}}$} \\
\hline Couple & 14 & 2 & 3 & \\
\hline Multiple generations & 34 & 5 & 18 & \\
\hline \multicolumn{5}{|l|}{ Exercise habit } \\
\hline Yes & 29 & 2 & 10 & \multirow[t]{2}{*}{ n.s. ${ }^{\mathrm{c}}$} \\
\hline No & 21 & 5 & 14 & \\
\hline The number of past medical history items & 3.0 & 2.0 & 3.0 & n.s. ${ }^{b}$ \\
\hline \multicolumn{5}{|l|}{ TMIG } \\
\hline Instrumental independence & 4.0 & $2.6(2.1)$ & 3.5 & \multirow[t]{3}{*}{ n.s. ${ }^{\mathrm{a}}$} \\
\hline Intellectual activity & 3.0 & $3.1(0.7)$ & 3.0 & \\
\hline Social role & 3.0 & $2.3(1.5)$ & 3.0 & \\
\hline \multicolumn{5}{|l|}{ Disease-specific QOL } \\
\hline Nonspecific conditions & 13.0 & 14.0 & $12.2(2.6)$ & \multirow[t]{3}{*}{ n.s. ${ }^{\mathrm{a}}$} \\
\hline Disease-specific conditions & 17.0 & $17.0(2.7)$ & 18.0 & \\
\hline Subjective indices & 25.0 & $19.3(6.3)$ & 24.0 & \\
\hline SF-36 & & & & n.s. ${ }^{\mathrm{a}}$ \\
\hline PCS & $39.9(8.8)$ & $44.2(4.6)$ & $41.8(6.3)$ & \\
\hline MCS & 59.6 & $52.2(8.5)$ & $53.5(8.1)$ & \\
\hline
\end{tabular}


Table 2 Correlation of disease-specific quality of life (QOL) scores and Short Form (36) Health Survey (SF-36) summary scores

\begin{tabular}{lll}
\hline Disease-specific QOL & SF-36 & \\
\cline { 2 - 3 } & PCS & MCS \\
\hline Nonspecific conditions & 0.01 & $0.51^{*}$ \\
Disease-specific conditions & 0.15 & $0.53^{*}$ \\
Subjective indices & 0.18 & $0.57^{*}$ \\
\hline
\end{tabular}

Values are expressed as Spearman's correlation coefficient

$* p<0.001$

patients and the control group consisting of 95 individuals. There were 63 men $(76.8 \%)$ and 19 women $(23.2 \%)$ in the cardiac surgery group and 27 men $(28.4 \%)$ in the control group. The median age was 72.5 (range 65-86) years in the cardiac surgery group and 77.0 (range 65-91) years in the control group. There were significant differences in gender ratio and age between groups $(p<0.001$ each): age was significantly lower in the cardiac surgery group. When comparing family composition and living with one's spouse, 64 patients $(78.0 \%)$ lived with their spouses, showing a higher rate than controls $(42,44.2 \%)$. Although the rate of living in a multigenerational family was high in both groups, the rate of only living with one's spouse was high in the cardiac surgery group, showing a significant difference between groups $(p<0.001)$. The number having an exercise habit was 41 parents $(50.0 \%)$ and 54 controls $(56.8 \%)$, showing a similar rate. Median number of past medical history items, including diseases currently under treatment, was 3.0 including heart disease in the cardiac surgery group whereas 15 controls $(15.8 \%)$ had no past medical history, and the mean number (1.0) was significantly lower $(p<0.001)$. Regarding TMIG, median scores for instrumental independence were 4.0 and 5.0 in the cardiac surgery and control groups, respectively; of intellectual activity 3.0 and 4.0, respectively; and of social role were 3.0 and 4.0, respectively. This showed that scores of all subscales were lower in the cardiac surgery than in the control group. When comparing SF-36 results, median PCS scores were 42.5 (range 17.8-62.2) and 44.4 (range 17.0-57.93) in the cardiac surgery and control groups, respectively, showing a lower score in the cardiac surgery group; however, MCS scores were 56.1 (range 34.1-69.6) and 55.3 (range 25.3-68.8), respectively, showing a lower score in the control group. As gender ratio and age were significantly different between groups, corrections for gender and age were applied. Table 3 shows, after corrections, the scores of all subscales of TMIG and PCS representing the physical health level, which was significantly lower in the cardiac surgery group $(p<0.001)$; no significant difference was noted in MCS representing the mental health level.
Table 3 Comparison of the Tokyo Metropolitan Institute of Gerontology (TMIG) and Short Form (36) Health Survey (SF-36) summary score between patients and the controls

\begin{tabular}{llll}
\hline & Patients & Controls & $p$ value \\
\hline TMIG & & & \\
Instrumental independence & 4.0 & 5.0 & $*$ \\
Intellectual activity & 3.0 & 4.0 & $* *$ \\
$\quad$ Social role & 3.0 & 4.0 & $*$ \\
SF-36 & & & \\
PCS & 42.5 & 44.4 & $* *$ \\
MCS & 56.1 & 55.3 & n.s. \\
\hline
\end{tabular}

The univariate analysis with age and sex

PCS physical component summaries, MCS mental component summaries, n.s. not significant

$* p<0.05, * * p<0.001$

Table 4 Comparison of disease types in past medical histories between patients and controls

\begin{tabular}{lccl}
\hline Previous diseases & $\begin{array}{c}\text { Patients } \\
\text { No. }(\%)\end{array}$ & $\begin{array}{c}\text { Controls } \\
\text { No. }(\%)\end{array}$ & $p$ values \\
\hline Hypertension & $47(57.3)$ & $30(31.6)$ & $* *$ \\
Disorder of bones, joints, muscles & $26(31.7)$ & $23(24.2)$ & n.s. \\
Diabetes mellitus & $21(25.6)$ & $10(10.5)$ & $*$ \\
Cerebrovascular disorders & $14(17.1)$ & $9(9.5)$ & n.s. \\
Heart diseases & $82(100)$ & $11(11.6)$ & $* * *$ \\
Respiratory diseases & $7(8.5)$ & $3(3.2)$ & n.s. \\
Others & $33(40.2)$ & $24(25.3)$ & $*$ \\
None & 0 & $15(15.8)$ & \\
\hline
\end{tabular}

Past medical history required multiple responses with Yes or No. Number of persons with disease history and being treated: values are expressed as Yes. Differences determined by Fisher's exact test or chi-square test

$* p<0.05, * * p<0.01, * * * p<0.001$

As shown by the comparison of associated factors between groups, the control group had fewer past medical histories than the cardiac surgery group despite their age being higher, showing their high QOL activity capacity and physical health level. At the same time, it was also clarified that there was no significant difference in mental health level between groups. Table 4 shows the disease types in the past medical histories of the two groups. Forty-seven patients (57.3\%) had hypertension in the cardiac surgery group, being the most frequent excluding heart disease, followed by bone/joint disease in $26(31.7 \%)$ and diabetes in $21(25.6 \%)$. In the control group, the frequency of hypertension was also the highest (30 individuals, $31.6 \%$ ), followed by bone/joint disease in $23(24.2 \%)$ and heart disease in $11(11.6 \%)$. High frequencies of hypertension and bone/joint disease were common in both groups, but those of hypertension $(p<0.01)$ and diabetes $(p<0.05)$ 
Table 5 Factors related to physical component summaries (PCS) of the Short Form (36) Health Survey (SF-36) score in patients and controls by multiple logistic regression analysis

\begin{tabular}{|c|c|c|c|c|c|c|c|c|}
\hline & \multicolumn{4}{|l|}{ Patients } & \multicolumn{4}{|l|}{ Controls } \\
\hline & \multicolumn{4}{|c|}{ Model $\chi^{2} 12.187, p<0.01$} & \multicolumn{4}{|c|}{ Model $\chi^{2} 35.118, p<0.001$} \\
\hline & \multicolumn{4}{|c|}{ Below (48) above (34) } & \multicolumn{4}{|c|}{ Below (48) above (47) } \\
\hline & $\beta$ & OR & $p$ & $95 \% \mathrm{CI}$ & $\beta$ & OR & $p$ & $95 \% \mathrm{CI}$ \\
\hline Age & -0.114 & 0.892 & $*$ & $0.805-0.989$ & & & & \\
\hline \multicolumn{9}{|l|}{ TMIG } \\
\hline \multicolumn{9}{|l|}{ Instrumental independence } \\
\hline \multicolumn{9}{|l|}{ Intellectual activity } \\
\hline Social role & & & & & 0.795 & 2.215 & $* *$ & $1.236-3.972$ \\
\hline MCS & -0.067 & 0.935 & $*$ & $0.883-0.990$ & -0.159 & 0.853 & $* * *$ & $0.793-0.910$ \\
\hline The number of past medical history items & & & & & -0.864 & 0.421 & $* *$ & $0.246-0.721$ \\
\hline
\end{tabular}

$O R$ odds ratio, $C I$ confidence interval, TMIG Tokyo Metropolitan Institute of Gerontology, MCS mental component summaries

Stepwise $* p<0.05, * * p<0.01, * * * p<0.001$

after excluding heart disease were significantly higher in the cardiac surgery group.

QOL-associated factors by group

To identify factors influencing differences in QOL score using the SF-36, the association with factors was investigated in each group. Groups were divided into high- and low-score subgroups according to PCS and MCS medians in the control group, and multiple logistic regression analysis was performed using the stepwise method. Explanatory variables were gender, age, three subscales of the TMIG index, number of past medical history items, exercise habit, living with or without one's spouse, and family composition; MCS was added in the analysis, with PCS as a target variable. Results are shown in Table 5. Regarding factors associated with PCS score level, age and MCS showed negative influences in the cardiac surgery group. In the control group, social role in the TMIG index was a positive factor; MCS and number of past medical history items were negative factors. A similar analysis was performed for MCS, but neither group showed a fit to this model.

\section{Discussion}

The study group consisted of elderly patients after cardiac surgery who primarily spent their lives independently at home. As a prolonged period had passed after surgery, and patients with poor postoperative outcomes were excluded, not all patients treated with cardiac surgery were represented. Because the number of patients was only 82 , insufficient to analyze by surgical procedure, no surgical- procedure-associated significant difference was noted in QOL. Comparison involving a sufficient number of patients is necessary to confirm this.

We compared QOL findings on the disease-specific scale measured in the cardiac surgery group with findings reported by Kobayashi et al. [18]. No significant difference was noted in the nonspecific health condition domain, but disease-specific symptoms score was significantly lower, and feelings of chest compression and symptoms of angina pectoris were severe in the cardiac surgery group. The study group reported by Kobayashi et al., comprised 21 and 22 (43) patients treated with coronary artery bypass and valve replacement, respectively; age was $60.6( \pm 8.8)$ years, and time after surgery was $22.4 \pm 24.0$ months, suggesting that differences in age and QOL measurement time point influenced the findings.

There has been no previous report showing differences in QOL-associated factors based on comparison with the general elderly population on the condition that MCS during the specified period may have been influenced by the disease-specific QOL in elderly cardiac surgery patients. Thus, we consider this was a significant study for investigating the influence of cardiac surgery on QOL. We examined relationships between disease-specific and overall QOL and found that the disease-specific QOL positively associated with MCS. Accordingly, it is suggested that resolution of disease-specific symptoms by cardiac surgery may increase MCS scores. In the control group, the elderly individuals were judged as capable of being interviewed by the medical student doing the interview and, accordingly, their health level was relatively high, which was problematic for regarding them as general elderly individuals. Therefore, biases in selection of the two groups could not be ruled out, and it is difficult to 
generalize the results of comparisons to all cardiacsurgery-treated and general elderly individuals, which is a limitation of this study.

When QOL employing SF-36 was compared between groups, the PCS score was significantly lower in the cardiac surgery group. MCS score among the groups did not differ, indicating that mental health level about 2 years after cardiac surgery and discharge was comparable with that of general elderly individuals. However, both PCS and MCS scores were significantly lower than the national standards [15] in both groups. This may have been because age was not considered in the national standards. When comparing QOL-associated factors, all TMIG index subscales were significantly lower in the cardiac surgery group and also significantly lower than the typical national values reported by Koyano et al. [17] (mean \pm SD, $10.8 \pm 3.0$ ). Regarding family composition, the rate of living with one's spouse was high in the cardiac surgery group, whereas the rate of living in a multigenerational family without living with one's spouse was a characteristic of the control group. Considering that the age was lower and the proportion of men was higher in the cardiac surgery than in the control group, it may have been possible that the low activity capacity and physical health levels were due to their dependence on their families. A marked difference was also noted in the number of past medical history items between groups. Despite the age being high (77 years), $15.8 \%$ of the control group had no past medical history, indicating a high health level. Regarding disease type of past medical histories, frequencies of diabetes and hypertension were higher in the cardiac surgery group, which was convincing, because these are underlying diseases associated with heart disease. Comparing QOL-associated factors between groups clarified different and common points. When the factors associated with PCS were compared, the differences were as follows: age was a negative factor in the cardiac surgery group: physical health level was high at a young age. In the control group, a high activity capacity and lower number of past medical history items positively influenced physical health level. The common point was the association of MCS in a negative direction.

TMIG and the number of past medical history items influenced PCS in the control group, which is convincing. Age was not included in the influential factors, which may have been due to achieving successful aging at a higher age (77 years) in the control than in the cardiac surgery group. Rowe and Kahn [19] identified three factors involved in successful aging: having habits associated with less risk factors for diseases, elevation of cognitive and physical functions in later life, and maintenance of social relationships and active attitudes in life by participating in productive activities. PCS-associated factors were high activity capacity and social role scores and a low number of past medical history items in the control group, indicating successful aging. In the cardiac surgery group, age was a factor determining PCS level, and physical health level worsened as age increased. Many studies on QOL comparison between before and after cardiac surgery paid attention to the age [20-23], and Saito et al. [24] reported age, gender, and presence or absence of community society were QOL-determining factors of myocardial infarction patients. Our findings support these reports. It seems that physical health in the patient group deteriorates with aging, and medical and nursing care are needed to maintain physical function among these patients.

As a factor common to both groups, MCS negatively influenced PCS. It suggests that irrespective of high MCS, PCS may be lowered, and that elevation of the physical health level with a decline in mental health level was consistent. In reality, elderly individuals with cardiac surgeries sometimes limit daily activities for fear of cardiac symptoms, and elderly individuals without cardiac diseases sometimes limit daily activities because of other diseasesor age-related deterioration. As a result, this leads to decreased MCS. Therefore, we it is important that those elderly individuals use their PCS well. As the elderly with high PCS — even after cardiac surgery — may have lowered MCS, physicians and nurses need to be aware of and attend to those considerations.

After submission of our study plan to and approval from the Ethics Committee of Fukui Prefectural Hospital, our objective was explained to patients when they visited the hospital, and written consent was obtained before the interview. Medical students orally and using documents explained the study to control participants, and those who consented were interviewed.

Acknowledgments We are grateful to Dr. Shinichiro Yamamoto, head cardiovascular surgeon of Fukui Prefectural Hospital (director of Fukui Prefectural Hospital) and nurses of the outpatient clinic for their understanding and support of this study, and patients at the cardiovascular surgery outpatient clinic of Fukui Prefectural Hospital for their cooperation in the survey.

\section{References}

1. Ministry of Health, Labour, Welfare. Major of cause of death in 2009. J Health Welf Stat 2010/2011. 2010;57:47-59.

2. Sezai Y. The coronary artery surgery results 2008. Japanese Association for Coronary Artery Surgery. http://www.jacas.org/ data/pdf/slide_2007.pdf. Accessed 30 June 2011.

3. Nishiyama S. A study of quality of life (QOL) in patients with ischemic heart disease. J Jpn Coron Assoc. 2004;10:74-8.

4. Kitamura H, Okabayashi H, Hanyu M, Nakano J, Nomoto T, Sakaguchi J, Ueno H, Matuo T. Early and late results and problem of coronary artery bypass grafting in patients over 80-years-old. Jpn J Thoracic Cardiovasc Surg. 2005;58:1035-7. 
5. Komesu I, Arinaga K, Nakazima A, Kozima Y, Kimura S, Ishihara K, Kawauchi Y. Early and mid-term survival and quality of life after thoracic aortic surgery in patients aged 70 years and older. Jpn J Cardiovasc Surg. 2001;30:177-81.

6. Fruitman DS, Macdougall CE, Ross DB. Cardiac surgery in octogenarians: Can elderly patients benefit? Quality of life after cardio surgery. Ann Thorac Surg. 1999;68:29-35.

7. Oldridge NB, Gottlieb M, Guyatt G, Jones N, Streiner D, Feeny D. Predictors of health-related quality of life with cardiac rehabilitation after acute myocardial infarction. J Cardiopulmonary Rehabil. 1998;17:95-103.

8. Oldridge NB. Outcome assessment in cardiac rehabilitation. J Cardiopulmonary Rehabil. 1197;17:179-94.

9. Oda K, Hata M, Kawatsu S, Adachi O, Yamaya K, Saiki Y, Sakurai M, Akasaka J, Iguchi A, Tabayashi K. Quality of life in elderly patients following thoracic aortic surgery. J Jpn Thorac Cardiovasc Surg. 2004;52:515-23.

10. Ogino H, Ueda Y, Sugita T, Sakakibara Y, Matsuyama K, Matsubayashi K, Nomoto T. Early and midterm outcomes of cardiac and thoracic aortic surgery in over-75-years-olds with postoperative quality of life assessment. J Jpn Thorac Cardiovasc Surg. 1999;47:57-62.

11. Li R, Watanabe T, Hosoda Y. Operation indication of coronary artery bypass grafting in elderly patients based on long-term results. J Jpn Assoc. 1999;5:102-5.

12. Fukuhara S, Suzukamo Y. Profiling of health-related QOL scale; focusing on SF-36. In: Ikegami N, Fukuhara S, Simozuma K, Ikeda S, editors. QOL evaluation handbook for clinical. Tokyo: Igaku-Shoin; 2001. p. 32-42.

13. Kayaba K, Nagashima K, Saito M, Omae T, Seki A, Arakawa K, Ishii T, Kameyama M, Kokufu T, Fujii J. Development of QOL evaluation method in circulatory organs disease treatment. J Jpn Assoc Cerebro cardiovasc Dis Control. 1990;25:89-95.

14. Yamamoto A, Yamaguchi T, Kangawa K, Kamiya T. Study on the management system for the aged patients with cardiovascular diseases to improve their quality of life. In: Fujii J, editor. Annual report of the research on cardiovascular diseases. Osaka: National Cardiovascular Center; 1992. p. 592-4.

15. Fukuhara S, Suzukamo Y, Bito S, Kurokawa K. Manual of SF-36. Japanese version 1.2. Tokyo: Public Health Research Foundation; 2001.

16. Ware JE, Gandek B. Overview of SF-36 health survey and the international quality of life assessment (IQOLA) project. J Clin Epidemiol. 1998;51:903-12.

17. Koyano W, Shibata H, Nakasato K, Hoga H. Measurement of competence in the elderly living at home: development of an index of competence. Jpn J Public Health. 1987;34:109-14.

18. Kobayashi M, Futawatari T, Hirose K, Seto M. The quality of life realities and associated factors in the patients who underwent cardiovascular surgery. Adult Nursing Society 2. Jpn Nursing Assoc. 1997;28:18-21.

19. Rowe JW, Kahn RL. Successful aging and disease prevention. Adv Renal Replace Ther. 2000;7:70-7.

20. Komesu I, Arinaga K, Nakashima A, Toshima Y, Oohashi M, Eto N. Early and mid-term results after cardiac or thoracic aortic surgery in patients aged 70 years and older: survival and quality of life. Jpn General Thoracic Cardiovasc Surg. 2001;54:457-62.

21. Mitsumaru A, Yozu R, Ueda T, Kawada S. Early and late results of cardiac and thoracic aortic surgery in patients older than 75 years from a quality of life point view. Jpn General Thoracic Cardiovasc Surg. 1997;50:718-21.

22. Shigemitsu O, Hadama T, Miyamoto S, Anai H. Early and longterm results of cardiovascular surgery in octogenerations. Ann Thorac Cardiovasc Surg. 2001;7:223-31.

23. Ura M, Sakata R, Umebayashi Y, Ueno T. Coronary artery bypass surgery in patients over 80 years. General Thoracic Cardiovasc Surg. 1996;44:1124-9.

24. Saito M, Katsuki T, Kayaba K. The quality of life and prescribe factors of myocardial infarction. Ther Res. 1990;11:2454-9. 